\title{
CLOSE APPROACH ASTROMETRY A Method for Improving Reference Systems
}

\author{
T. NAKAMURA, H. SHIBASAKI \\ National Astronomical Observatory \\ Osawa, Mitaka, Tokyo 181 JAPAN
}

\begin{abstract}
For improved establishment of the dynamical reference frame and tying it to the stellar reference frame, a method to observe close approach (CA) events between satellite and satellite and/or satellite and star is proposed here. The accuracy of measurements of angular distance is $0 " .02$ - 0 ". 03 for a single data point. We apply this technique fairly successfully to the CA events of the Galilean satellites and a preliminary orbital longitude correction to the E2X3 constants of J4 is obtained from eight CA observations ranging from 1987 to 1989.
\end{abstract}

\section{Introduction}

For high-precision observations of planetary positions, two technical improvements were recently proposed. The first is to observe the positions of the satellites, rather than planet itself (e.g., Pascu and Schmidt, 1990). This is because diameters of satellites are small and thus their phase effects are negligible. The second is to use intersatellite observations; (e.g., Taylor and Shen, 1988); this has the advantage that possible systematic errors of the adopted catalogue for plate solution can be eliminated.

If this line of improvement is pursued for the purpose of establishing the dynamical reference frame and tying it to the stellar reference frame, the following two step procedures will apply: high-precision intersatellite observations and satellite-to-star observations. Once a high-precision ephemeris of the satellite is available, determination of the right ascension and declination of stars based on the dynamical reference frame is fairly easy through measurements of CA events between the stars and the satellite. Therefore we restrict ourselves to high-precision measurements of satellites positions in this paper.

It is well known that, due to the strong correlation of turbulent motions of adjacent air masses, an apparent angular distance of two close celestial bodies can be determined much more accurately than that between telescope's cross threads and a single object. Lindegren (1980) predicts that 0".03-0".04 accuracy is achieved for a relative distance of 30 "-40" with a time integration of $20 \mathrm{sec}$. Our experience in double star observations gives a little better accuracy than Lindegren's theory. Han (1989) also reports a few times better accuracy than Lindegren's formula.

In this paper this advantage of short distance measurements was applied to the observations of the CA events of the Galilean satellites and the results were compared with the SampsonLieske theory. From the observations of the events including J4, a preliminary orbital longitude correction was determined. As far as we know, there seems to be no such applications of this 
technique yet to practical observations of satellites, though the similar ideas have been proposed (e.g., Kammeyer et al., 1990).

\section{Prediction of close approach events}

For observations of any CA events, prediction calculations are essential. The CA events of the Galilean satellites were calculated by M. Soma using GALSAP program provided by J.H. Lieske and it is found that the CAs of less than 30" take place as many as 60-100 times every year at an observing site. This is due to the ecliptic plane being close to the orbital planes of the Galilean satellites.

\section{Observations and data reduction}

A telescope of long focal length is essential to achieve high accuracy measurements. Observations were made at the Cassegrain focus $(\mathrm{f}=16.5 \mathrm{~m})$ of a 36 -inch reflector (Dodaira station, Japan) with a CCD-type TV camera (field of view: 110"x83") and the images were recorded in a SONY U-matic ( $2 / 3$ inch) video recorder, with time signals synchronized to UTC up to about 10 microsec. The play-backed image signals were time-integrated on a frame memory as digital data of pixel size of 14 micron square which corresponds to 0 ".16 square on the sky. In order to determine scale value, we observed the diurnal motion of Polaris by stopping the sidereal drive of the telescope and compared the measured lengths of Polaris' arc with the daily positions of the star given in the Japanese Ephemeris. We also sometimes observed a double star Sigma CAM 1694 to check Affin geometry of pixel spacing of the CCD chip used. A Gaussian profile with a quadratic- polynomial background was fitted to the marginal brightness distribution of star images. After ordinary flat- fielding, centroiding accuracy of $1 / 10$ pixels is easily realized. Typical integration time was $30 \mathrm{sec}$ (900 frames) for the satellites and $20 \mathrm{sec}$ for Polaris.

Twenty two CAs of less than about 60" were observed from October 1987 through March 1989. Observation time of an event ranges from $1 \mathrm{hr}$ to $3.5 \mathrm{hr}$ depending on the circumstance of each CA, nearly centered at the closest approach time in most cases. From these data several frames of $30 \mathrm{sec}$ integration were obtained at the interval of 10-15 min.

The relative distance $d$ of two close satellites 1 and 2 can be written, with enough accuracy, as

$$
d=\left(q^{2}+p^{2} \cos ^{2} \delta_{1}-p^{2} q \sin \delta_{1} \cos \delta_{1}\right)^{1 / 2}
$$

where $p=\alpha_{2}-\alpha_{1}$, and $q=\delta_{2}-\delta_{1}$. Then the difference between observed and calculated distance $\Delta d$ is expressed using the errors in $\alpha$ and $\delta$ as follows:

$$
\Delta d=p / d \cos ^{2} \delta_{1} \Delta p+q / d \Delta q
$$

where $\Delta p=\Delta \alpha_{2}-\Delta \alpha_{1}$, and $\Delta q=\Delta \delta_{2}-\Delta \delta_{1}$. Since $\Delta \alpha$ and $\Delta \delta$ in equation (2) can be connected through GALSAP program to the corrections to the Lieske's orbital parameters $\epsilon_{i}$ and $\beta_{i}$ (Lieske, 1980), we adopt equation (2) as an observation equation for orbital improvement.

If we wish to compare observations with theory at the error level of say 0 ". 005 , various small corrections to the observed distance are necessary. Among them the effects due to differential refraction are most important. Since refraction is larger for shorter wave length, the observations without filter or with a broad-band filter cause stellar images to elongate in the altitude 
direction, especially for large zenith distances; this is called the atmospheric prism effect. This effect is troublesome because the surface brightness distribution in the elongated images depends on both the wave-length sensitivity of the detector system used and color of the object observed. However, our model calculations showed that, for zenith distance of less than $75^{\circ}$ and relative distance of less than a few arcmin in the altitude direction, correction of monochromatic differential refraction is sufficient as far as the error level of 0 ". 005 is allowed.

\section{Preliminary longitude correction}

An example of the time variation of $\Delta d\left(=d_{o b s}-d_{c a l}\right)$ is given in Fig.1. In actual solutions of equation (2), the correction to scale value $(f)$ had to be incorporated as a form of $d_{c a l} \Delta f / f$. This is probably because Jupiter was in the southern sky whereas Polaris in the northern sky and thus the atmospheric effect on them was different.

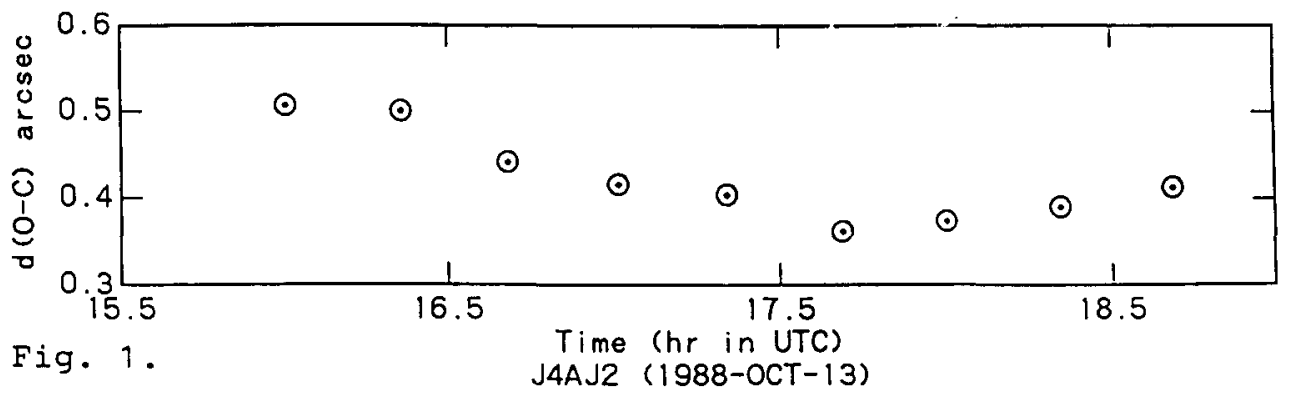

For a preliminary orbital analysis, we assume that only the mean orbital longitudes $\left(\beta_{1}, \beta_{2}\right.$ and $\beta_{4}$ ) of the satellites need improvement. $\partial d / \partial \beta$ is largest at the inferior conjunction of the satellites with Jupiter and zero near elongation. In case of the CA events involving J4, the relevant inner satellite tends to be near elongation. This means that unless the longitude errors of the satellites other than J4 are unusually large (this is actually the case for the Galilean satellites), $\partial d / \partial \beta_{4} \Delta \beta_{4}$ is much larger than other terms in equation (2). Actually in most of our observations involving $\mathrm{J} 4, \partial d / \partial \beta_{4}$ is proved to be 10 to a few 10 times larger than other derivatives. Moreover, in the mutual events, the phenomena involving $\mathrm{J} 4$ are least observed ones (e.g., Aksnes and Franklin, 1976). Therefore we have tried to obtain a preliminary correction to the E2X 3 constants of J4 using eight J4 CA events whose partner is $\mathrm{J} 1$ or J2. We have neglected $\Delta \beta_{1}$ or $\Delta \beta_{2}$ because of the reason mentioned above. Since $\Delta \beta_{4}$ and $\Delta f / f$ were not correlated each other during our observation period because of the Earth being far from the orbital planes of the satellites, we could solve $\Delta \beta_{4}$ from each single event. Then we have $-0^{\circ} .027 \pm 0^{\circ} .021$ for the averaged $\Delta \beta_{4}$. An overall solution is now being undertaken by using all the observed events and by taking account of other errors of orbital elements.

Finally we emphasize that CA events will have wide applications in Saturnian and Uranian satellites, asteroid-asteroid, asteroid-star, satellite-star, and so on.

\section{References}

Aksnes,K.,Franklin,F.A.:1976, Astron. J. 11 , 464.

Han,I.:1989, Astron. J. $\underline{97}, 607$. 
Kammeyer,P.C.,Fliegel,H.F.,Schmidt,R.E.,Harrington,R.S.:1990, this Colloquium.

Lieske,J.H.:1980, Astron. Astrophys. 82, 340.

Lindegren,L.:1980, Astron. Astrophys. $\underline{89}, 41$.

Pascu,D.,Schmidt,R.E.:1990, Astron. J. 99, 1974.

Taylor,D.B.,Shen,K.X.:1988, Astron. Astrophys. 200, 269. 